\title{
Absence of self-averaging in the complex admittance for transport through random media
}

\author{
Mitsuhiro Kawasaki and Takashi Odagaki \\ Department of Physics, Kyushu University, Fukuoka 812-8581, Japan \\ Klaus W. Kehr \\ Institut für Festkörperforschung, Forschungszentrum Jülich GmbH, D-52425 Jülich, Germany
}

(Received 3 June 1999; revised manuscript received 15 November 1999)

\begin{abstract}
A random walk model in a one-dimensional disordered medium with an oscillatory input current is presented as a generic model of boundary perturbation methods to investigate properties of a transport process in a disordered medium. It is rigorously shown that an admittance which is equal to the Fourier-Laplace transform of the first-passage time distribution is non-self-averaging when the disorder is strong. The low-frequency behavior of the disorder-averaged admittance, $\langle\chi\rangle-1 \sim \omega^{\mu}$, where $\mu<1$, does not coincide with the lowfrequency behavior of the admittance for any sample, $\chi-1 \sim \omega$. It implies that the Cole-Cole plot of $\langle\chi\rangle$ appears at a different position from the Cole-Cole plots of $\chi$ of any sample. These results are confirmed by Monte Carlo simulations.
\end{abstract}

Response of a system to an external field is a standard information to be utilized in the study of condensed matter. Recently, the frequency response method ${ }^{1}$ and the intensity modulated photocurrent spectroscopy ${ }^{2}$ have been introduced, where an oscillatory perturbation is applied at one end of a system and a response from the other end of the system is measured. Thus, these experimental techniques can be considered to belong to a generic method which can be called the boundary perturbation method (BPM). In the presence of a periodically forced boundary condition on one end of a system, the output from the other end of the system is in proportion to the perturbation in the linear regime and the proportionality constant is called the admittance. The frequency dependence of the admittance contains various information of the dynamics of the system. The BPM is expected to provide useful information on transport properties of disordered media.

The analysis of transport of particles in a random medium attracts great interest since the transport mechanism is basic for the understanding of many physical phenomena, from electrical conductivity to thermal properties. ${ }^{3}$ Usually it is assumed that a sample used in experiments is sufficiently large that it can be considered to be composed of a large number of subsystems. If each of the subsystems is itself macroscopic, the boundary effect is negligible and each subsystem may be considered to be a realization of the system with a particular choice for the disorder. Thus, a measurement of any observable in such a system corresponds to an average over all the subsystems, i.e., an average over the ensemble of all realizations of the disorder. Systems for which this assumption is valid are said to be self-averaging (SA). Since the assumption implies no sample dependence, it guarantees reproducibility of experimental results in any sample.

However, it is known that the disorder-averaged quantity follows a dynamics that is different from the dynamics of the quantity of each sample. If the dynamics of particles in a disordered sample is assumed to be stochastic and Markovian, i.e., to follow a master equation, the disorder-averaged master equation has a memory effect. ${ }^{4}$ Thus, the dynamics of an averaged quantity may be non-Markovian even though the process for each sample is Markovian. There are several reports on absence of SA in transport phenomena in disordered media: for mean square displacement, ${ }^{5}$ for mean firstpassage time of the Sinai model. ${ }^{6}$

In this report, we study a random walk model in a onedimensional disordered medium with an oscillatory input current as a generic model of the BPM. It is rigorously shown that the admittance, which is equal to the FourierLaplace transform of the first-passage time distribution (FPTD), is non-self-averaging (non-SA) when the disorder is strong.

We consider a general random walk in a one-dimensional lattice segment of $N+1$ sites. The lattice sites are denoted by integers, $n=0,1, \ldots, N$. The dynamics of a random walking particle can be described by a master equation for the probability $P_{n}(t)$ that the particle is at site $n$ at time $t \geqslant 0$. The master equation for the model is written as

$$
\begin{aligned}
\dot{P}_{n}(t)= & w_{n n-1} P_{n-1}(t)-\left(w_{n-1 n}+w_{n+1 n}\right) P_{n}(t) \\
& +w_{n n+1} P_{n+1}(t),
\end{aligned}
$$

where $w_{m n}$ denotes the random jump rate of a particle from site $n$ to site $m$. The probability distribution of jump rates characterizes the random medium. We introduce a perturbation at the left end, so that the equation for $P_{0}(t)$ is given by

$$
\dot{P}_{0}(t)=-w_{10} P_{0}(t)+w_{0}{ }_{1} P_{1}(t)+J(t) .
$$

$J(t)$ is the oscillatory current perturbation at site 0 . The right end of the system is assumed to be a sink and the equation for $P_{N-1}$ is given by

$$
\begin{aligned}
\dot{P}_{N-1}(t)= & w_{N-1 N-2} P_{N-2}(t) \\
& -\left(w_{N-2 N-1}+w_{N N-1}\right) P_{N-1}(t) .
\end{aligned}
$$

Since the current perturbation $J(t)$ oscillates in time around a positive average with the amplitude $\Delta J$, the response of the 
output current $w_{N N-1} P_{N-1}(t)$ oscillates around its stationary state with the amplitude $w_{N N-1} \Delta P_{N-1}$ at the same frequency with a phase-shift. The admittance is defined as the ratio of these two amplitudes

$$
\chi(\omega) \equiv \frac{w_{N N-1} \Delta P_{N-1}}{\Delta J} .
$$

We first note that the admittance can be related to the first passage time distribution $F_{N 0}(t)$ (FPTD), which is the probability density that the particle which starts at site 0 at time 0 arrives for the first time at site $N$ at time $t$. Since site $N$ is a sink, the FPTD is given by the output current from site $N$ when there is no input current and the particle starts from site 0 at time 0 . The Fourier-Laplace transform of a system of master equations for the case is the same as a system of equations for $\Delta P_{n} / \Delta J$ derived from the master equations Eqs. (1)-(3). It implies that $\widetilde{F}_{N 0}(s=i \omega)$ for the case where there is no input current and the particle starts from site 0 at time 0 is identical to $w_{N N-1} \Delta P_{N-1} / \Delta J$. Thus, the admittance is equal to the Fourier-Laplace transform of the output current in the problem described above, i.e., the FPTD.

We make the low-frequency expansion of the admittance to see the behavior near the static limit. Since the admittance is given by the Fourier-Laplace transform of the FPTD, the admittance at zero frequency equals one due to normalization of the FPTD. The mean first-passage time (MFPT) $\bar{t}$ which is the first moment of the FPTD is given by $\bar{t}$ $=i d \chi(\omega=0) / d \omega$. Thus, the low-frequency expansion of the admittance is given by

$$
\chi(\omega)=1-i \bar{t} \omega+O\left(\omega^{2}\right) .
$$

Since the MFPT is given by ${ }^{7}$

$$
\bar{t}=\sum_{k=0}^{N-1} \frac{1}{w_{k-1} k}+\sum_{k=0}^{N-2} \frac{1}{w_{k+1}} \sum_{k}^{N-1} \prod_{j=k+1}^{i} \frac{w_{j-1 j}}{w_{j+1} j},
$$

the MFPT $\bar{t}$ is larger than $\sum_{k=0}^{N-1} 1 / w_{k-1 k}$. By taking the average of Eq. (6) over the distribution for the jump rates, one sees that the average of the MFPT $\langle\bar{t}\rangle$ is larger than the first inverse moment of a random jump rate $\langle 1 / w\rangle$. Thus, when the first inverse moment of a jump rate diverges, which is called strong disorder, the disorder-averaged MFPT diverges. It indicates that the disorder-averaged admittance is non-analytic. Since the admittance is a function of $i \omega$, we can write as

$$
\langle\chi(\omega)\rangle-1 \sim(i \omega)^{\mu},
$$

where $0<\mu<1$. Since this behavior of the disorderaveraged admittance is completely different from the behavior of the admittance of each sample given by Eq. (5), the admittance is non-SA when the disorder is strong.

On the other hand, when the first inverse moment of a jump rate exists, it is easily shown from Eq. (6), that the MFPT is finite provided that there is no correlation between $w_{k+1 k}$ and $w_{i+1 i}$, where $i \geqslant k+1$. The condition of independence of the neighboring jump rates holds for the site disordered model called random trap model and the bond disordered model called a random barrier model. Thus, when

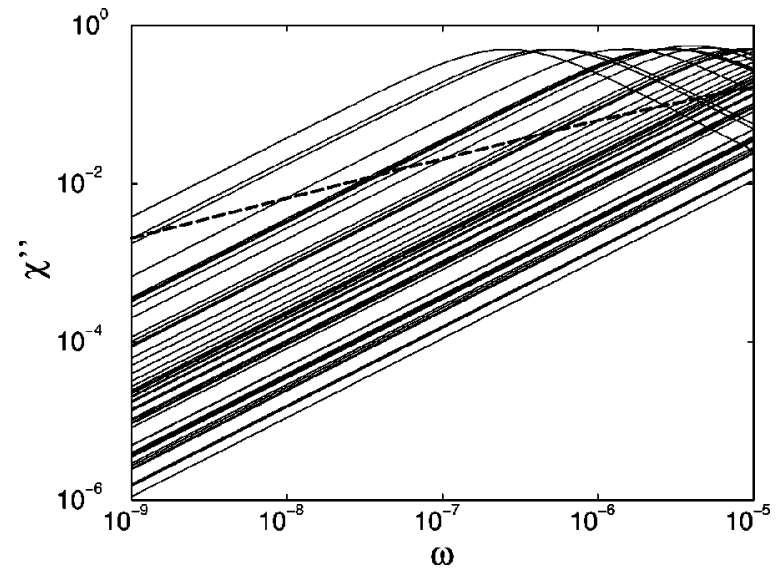

FIG. 1. Low-frequency behavior of the imaginary part of the admittance when the exponent of the probability distribution of jump rates is $1 / 2$ and the length of a chain is 100. Each solid line represents the admittance for each of 50 samples. The dashed line represents the admittance averaged over 5000 samples. Though the admittance of each sample is proportional to $\omega$, the averaged admittance is proportional to $\sqrt{\omega}$.

the first inverse moment of a jump rate exists, the lowfrequency behavior of the disorder-averaged admittance is the same as the one of each sample given by $\chi-1 \sim i \omega$. Furthermore, if the second inverse moment of a jump rate exists, the prefactor of $\omega$ does not depend on a realization of the chain when the chain is sufficiently long.

In order to test the foregoing observation, we performed a computer simulation in one-dimensional chain of 100 sites. Figure 1 shows the low-frequency behavior of the imaginary part of the admittance obtained by the Monte-Carlo simulation where the relation $w_{i+1 i}=w_{i+1}$ is assumed and the probability distribution of jump rates is given by

$$
P(w)=\left\{\begin{array}{l}
\alpha w^{\alpha-1} \quad \text { if } 0<w<1, \\
0 \quad \text { otherwise }
\end{array}\right.
$$

where $0<\alpha<1$. The power-law probability distribution corresponds to the waiting-time distribution $\Psi(t) \sim t^{-\alpha-1}$ used in the study of dispersive transport where $\alpha$ is proportional to temperature, ${ }^{8}$ and it has been shown that the power-law distribution is common for activation processes with random activation energy. ${ }^{9}$

In the literature of experiments of the BPM, ${ }^{1,2}$ the ColeCole plot of the admittance is employed for the analysis. It is a parametric plot of the imaginary part of the admittance against the real part. The shape of the Cole-Cole plot is independent of the length of the chain for a sufficiently long chain due to time-scale invariance of the Cole-Cole plot. Thus, the Cole-Cole plot shows the size-independent characteristics of a medium.

In order to see the non-SA property of the admittance in the Cole-Cole plot, we first note that the inverse of the admittance satisfies the following recursive relation:

$$
\begin{aligned}
\chi_{n}^{-1}(\omega)= & \left(\frac{i \omega}{w_{n+1 n}}+1+\frac{w_{n-1 n}}{w_{n+1 n}}\right) \chi_{n-1}^{-1}(\omega) \\
& -\frac{w_{n-1 n}}{w_{n+1 n}} \chi_{n-2}^{-1}(\omega),
\end{aligned}
$$




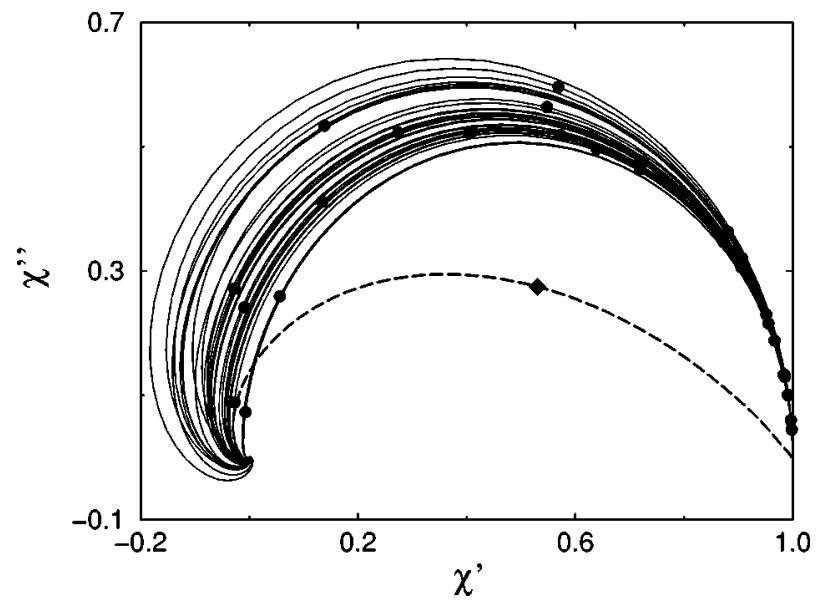

FIG. 2. The Cole-Cole plot of the admittance when the exponent of the probability distribution of jump rates is $1 / 2$ and $N=100$. Each solid line represents the admittance for each of 30 samples. The dashed line represents the admittance averaged over 5000 samples. The circles represents the admittance of each sample at $\omega=5 \times 10^{-7}$. Although the circles scatter outside the Debye semicircle, the diamond, i.e., the averaged admittance is inside of the Debye semicircle.

where $\chi_{n}^{-1}(\omega)$ is the inverse of the admittance for a chain of length $n .{ }^{10}$ The recursive relation proves inductively that the inverse of the admittance obeys the following four inequalities in the frequency region $\left[0, \omega_{n}\right]$, where $\omega_{n}$ is the positive smallest zero of $\chi_{n}^{-1^{\prime}}$ :

$$
\begin{aligned}
& \chi_{n}^{-1^{\prime \prime}}>0, \\
& \chi_{n-1}^{-1^{\prime}}>0, \\
& \chi_{n}^{-1^{\prime \prime}}>\chi_{n-1}^{-1^{\prime \prime}}, \\
& \chi_{n}^{-1^{\prime}}<\chi_{n-1}^{-1^{\prime}} .
\end{aligned}
$$

Since the second inequality shows $\omega_{n} \leqslant \omega_{n-1}$, the fourth inequality implies $\chi_{n}^{-1^{\prime}}<\chi_{n-1}^{-1^{\prime}}<\cdots<\chi_{1}^{-1^{\prime}}=1$ in the region $\left[0, \omega_{n}\right]$. It is straightforward to show that $\chi^{-1^{\prime}}<1$ implies that the Cole-Cole plot of the admittance of each sample can exist only outside a semicircle whose center is at $(1 / 2,0)$ and radius is $1 / 2$, which we call the Debye semicircle, when $\omega$ $<\omega_{n}$.

On the other hand, we can show in the following way that the Cole-Cole plot of the averaged admittance is located inside the Debye semicircle when the disorder is strong. We consider the angle $\theta$ between the tangent of the Cole-Cole plot at $(1,0)$ and the horizontal axis. For Eq. (7), $\theta=\mu \pi / 2$, which is less than $\pi / 2$ since $\mu<1$, is obtained. However, the angle $\theta$ for the Debye semicircle is equal to $\pi / 2$. It implies that the Cole-Cole plot of the averaged admittance appears inside the Debye semicircle when the disorder is strong.
Since the Cole-Cole plot of the admittance of any sample appears outside the Debye semicircle, the Cole-Cole plot clearly shows non-SA property of the admittance. Figure 2 shows the Cole-Cole plot of $\chi$ and $\langle\chi\rangle$ obtained by Monte Carlo simulation when $\alpha=1 / 2$ in Eq. (8). Since the admittance of each sample at the same frequency scatters outside the Debye semicircle, the admittance averaged at the same frequency is inside the Debye semicircle. In the case that the first inverse moment of a jump rate exists, the angle $\theta$ of the Cole-Cole plot of the averaged admittance is $\pi / 2$, which is the same as the Cole-Cole plot of each sample, because of the regular behavior as $\langle\chi\rangle-1 \sim i \omega$.

In conclusion, we have presented a generic stochastic dynamical model in one-dimensional medium of the BPM and have shown that the admittance has important information about transport in disordered media, e.g., the FPTD. Thus the BPM will be a powerful technique to investigate the dynamical process in random media. We have also shown that the admittance is non-SA when the disorder is strong. Since the absence of SA is due to nonanalyticity introduced by an average over infinite number of samples, the behavior of the averaged admittance over infinite number of samples given by a theoretical analysis does not coincide with that of the admittance observed in experiments.

Non-SA behavior, i.e., strong sample to sample dependence may be observed in other experiments. For example, it is known that anomalous system-size dependence of the mobility, $\mu \sim N^{1-1 / \alpha}$, is observed in the time-of-flight experiment of amorphous semiconductors. ${ }^{8}$ The anomalous system-size dependence is due to the fact that the smallest value of random jump rates depends on the length of a chain. ${ }^{11}$ Since the smallest value dominates the mobility, the mobility is also a random variable. Thus, the anomalous system-size dependence implies absence of SA.

It is important to note that the system treated in the present report is purely one dimensional and it is still an open problem whether the admittance for higher-dimensional media are SA. Since one can consider a diffusing particle's path a one-dimensional chain and a higher-dimensional medium can be regarded as a bundle of many realizations of a particle's path, the total output from the medium is given by the sum of one-dimensional currents. Thus, the admittance for such a system may be SA, since the number of paths is very large when the medium is macroscopic. Thus, in order to see the non-SA behavior described here one needs to work in one-dimensional system or the case where the translational invariance is violated in only one direction since there is still the possibility that the SA assumption is valid for twoor three-dimensional systems.

This work was supported in part by Grant-in-Aid from the Ministry of Education, Science, Sports and Culture. One of us (M.K.) is thankful to the Institut für Festkörperforschung, Forschungszentrum Jülich for its hospitality, where part of the present work was done. 
${ }^{1}$ Y. Yasuda, K. Iwai, and K. Takakura, J. Phys. Chem. 99, 17852 (1995); Y. Yasuda, Heterog. Chem. Rev. 1, 103 (1994).

${ }^{2}$ P.E. de Jongh and D. Vanmaekelbergh, Phys. Rev. Lett. 77, 3427 (1996); G. Franco, J. Gehring, L.M. Peter, E.A. Ponomarev, and I. Uhlendorf, J. Phys. Chem. B 103, 692 (1999).

${ }^{3}$ S. Alexander, J. Bernasconi, W.R. Schneider, and R. Orbach, Rev. Mod. Phys. 53, 175 (1981); J.W. Haus and K.W. Kehr, Phys. Rep. 150, 263 (1987); G.H. Weiss and R.J. Rubin, Adv. Chem. Phys. 52, 363 (1983); J.P. Bouchaud and A. Georges, Phys. Rep. 195, 127 (1990).

${ }^{4}$ J. Klafter and R. Silbey, Phys. Rev. Lett. 44, 55 (1980).

${ }^{5}$ J.M. López, M.A. Rodríguez, and L. Pesquera, Phys. Rev. E 51, R1637 (1995).
${ }^{6}$ S.H. Noskowicz and I. Goldhirsch, Phys. Rev. Lett. 61, 500 (1988).

${ }^{7}$ K.P.N. Murthy and K.W. Kehr, Phys. Rev. A 40, 2082 (1989).

${ }^{8}$ G. Pfister and H. Scher, Adv. Phys. 27, 747 (1978).

${ }^{9}$ T. Odagaki, Phys. Rev. Lett. 75, 3701 (1995).

${ }^{10}$ The recursive Eq. (9) is derived from the following two equations for the FPTD obtained by its definition: $\widetilde{F}_{i+1 i}(s)=\widetilde{\psi}_{i+1}(s)$ $+\widetilde{\psi}_{i-1 i}(s) \widetilde{F}_{i+1 i-1}(s)$ and $\widetilde{F}_{i+1 i-1}(s)=\widetilde{F}_{i+1 i}(s) \widetilde{F}_{i i-1}(s)$, where $\widetilde{F}_{i j}(s)$ denotes the Laplace transform of the FTPD from site $j$ to site $i$ and $\widetilde{\psi}_{i j}(s)$ denotes the Laplace transform of the waiting time distribution for the jump from site $j$ to site $i$.

${ }^{11}$ K.W. Kehr, K.P.N. Murthy, and H. Ambye, Physica A 253, 9 (1998). 